\title{
Diagonal Minkowski classes, zonoid equivalence, and stable laws
}

\author{
Ilya Molchanov, Felix Nagel \\ Institute of Mathematical Statistics and Actuarial Science, \\ University of Berne
}

\begin{abstract}
We consider the family of convex bodies obtained from an origin symmetric convex body $K$ by multiplication with diagonal matrices, by forming Minkowski sums of the transformed sets, and by taking limits in the Hausdorff metric. Support functions of these convex bodies arise by an integral transform of measures on the family of diagonal matrices, equivalently, on Euclidean space, which we call $K$-transform. In the special case, if $K$ is a segment not lying on any coordinate hyperplane, one obtains the family of zonoids and the cosine transform. In this case two facts are known: the vector space generated by support functions of zonoids is dense in the family of support functions of origin symmetric convex bodies; and the cosine transform is injective. We show that these two properties are equivalent for general $K$.

For $K$ being a generalised zonoid, we determine conditions that ensure the injectivity of the $K$-transform. Relations to mixed volumes and to a geometric description of one-sided stable laws are discussed. The later probabilistic application gives rise to a family of convex bodies obtained as limits of sums of diagonally scaled $\ell_{p}$-balls.

Keywords: cosine transform; diagonal transformation; Minkowski class; stable law; zonoid

MSC(2010) Classification: 52A21, 52A22, 60D05, 60E07
\end{abstract}

\section{Introduction}

\subsection{Geometric background}

Typical transformations applied to convex bodies (non-empty convex compact sets) in Euclidean space are scaling, translation and rotation, or the whole group of invertible linear transformations. Considerably less is known about the case when convex bodies are transformed by actions of diagonal matrices, subsequently called diagonal transformations.

If $K$ is an origin symmetric segment which does not lie on a coordinate hyperplane, then the family of convex bodies generated by diagonal transformations applied to $K$ is the same as the family generated by rotations and scaling. For most other convex bodies $K$, the two generated classes are not the same. While diagonal transformations might 
seem too much dependent on the choice of the coordinate system, their use is further motivated by a probabilistic interpretation described below.

For two vectors $x, y \in \mathbb{R}^{n}$,

$$
x y=\left(x_{1} y_{1}, \ldots, x_{n} y_{n}\right)
$$

denotes their Hadamard (componentwise) product; for a convex body $K$ and any $u \in \mathbb{R}^{n}$,

$$
u K=\{u x: x \in K\}
$$

denotes $K$ transformed by the diagonal matrix with the diagonal elements given by $u$. The Minkowski sum of two compact sets $K$ and $L$ is $K+L=\{x+y: x \in K, y \in L\}$. The support function of a compact set $K$ is defined by

$$
h(K, u)=\sup \{\langle u, x\rangle: x \in K\}, \quad u \in \mathbb{R}^{n},
$$

where $\langle\cdot, \cdot\rangle$ denotes the inner product in $\mathbb{R}^{n}$. In this paper we consider convex bodies that are symmetric, which is always understood with respect to the origin.

Taking sums of diagonally transformed $K$ we obtain convex bodies

$$
c_{1} v_{1} K+\cdots+c_{m} v_{m} K
$$

for non-negative $c_{1}, \ldots, c_{m}$ and $v_{1}, \ldots, v_{m}$ from the unit sphere. Passing to the limit, we arrive at the family of convex bodies with support functions given by integrals of $h(v K, u)$ with respect to a finite measure $\mu$ on the unit sphere $\mathbb{S}^{n-1}$. These convex bodies are called diagonal bodies generated by $K$. Further, for signed measures $\mu$ on $\mathbb{S}^{n-1}$, consider the map

$$
\mu \mapsto\left(T_{K} \mu\right)(u)=\int_{\mathbb{S}^{n-1}} h(v K, u) \mu(d v),
$$

which we call the $K$-transform of $\mu$. The central topic of this paper is the richness of the family of sets given by (1.1), shown to be closely related to the injectivity property of the $K$-transform.

Each symmetric segment with end-points $-u$ and $u$ is obtained as the diagonal transformation $u I$ of the segment

$$
I=[-(1, \ldots, 1),(1, \ldots, 1)]
$$

with the end-points $(-1, \ldots,-1)$ and $(1, \ldots, 1)$. Thus, applied to $I$, the diagonal transformations yield the same family of sets as scalings and rotations. Recall that $h(v I, u)=$ $|\langle u, v\rangle|$. If $\mu$ is a measure, $T_{I} \mu$ is the support function of a zonoid, see [13, Th. 3.5.3] and [15]. Generalised zonoids are defined by their support functions $T_{I} \nu$ for signed measures $\nu$, more precisely, $K$ is a generalised zonoid if

$$
h(K, u)=\int_{\mathbb{S}^{n-1}}|\langle u, v\rangle| \nu(d v), \quad u \in \mathbb{R}^{n}
$$

where $\nu$ is a finite signed measure on $\mathbb{S}^{n-1}$ called the representing measure of $K$.

Consider the family of convex bodies with support functions $T_{K} \mu$ for (non-negative) finite measures $\mu$. This family is the smallest Minkowski class that contains $K$ and is invariant under diagonal transformations; we call it the diagonal Minkowski class generated 
by $K$. If diagonal transformations are replaced by general invertible linear transformations and $K$ belongs to a certain family $\mathscr{A}$ of convex bodies, the generated $\mathrm{GL}_{n}(\mathbb{R})$ invariant Minkowski class has been considered in [1]; its elements are called $\mathscr{A}$-bodies. Restriction to the group of rotations yields a rotation invariant Minkowski class, see [14]. The central question in [1, 14] concerns the richness of the $\mathrm{GL}_{n}(\mathbb{R})$-invariant or rotation invariant Minkowski classes.

\subsection{Probabilistic background}

From the probabilistic standpoint, the paper aims to determine which information on integrable random vectors is available from the expected values of certain sets of onehomogeneous even functions applied to them. A function $f: \mathbb{R}^{n} \mapsto \mathbb{R}_{+}$is said to be one-homogeneous if $f(c x)=c f(x)$ for all $c \geq 0$ and $x \in \mathbb{R}^{n}$. A major result in this direction is the following theorem from 9 .

Theorem 1.1. Let $\xi$ and $\eta$ be two integrable random vectors in $\mathbb{R}^{n}$. Then

$$
\mathbf{E} f(\xi)=\mathbf{E} f(\eta)
$$

for all measurable one-homogeneous even functions $f: \mathbb{R}^{n} \mapsto \mathbb{R}_{+}$if and only if

$$
\mathbf{E}|\langle u, \xi\rangle|=\mathbf{E}|\langle u, \eta\rangle|, \quad u \in \mathbb{R}^{n} .
$$

It is obvious that (1.5) implies (1.6); the inverse implication relies on the injectivity of the cosine transform

$$
\mu \mapsto \int_{\mathbb{S}^{n-1}}|\langle u, v\rangle| \mu(d v),
$$

which maps an even signed finite measure $\mu$ on the unit sphere $\mathbb{S}^{n-1}$ to a homogenous function of $u \in \mathbb{R}^{n}$, see [13, Sec. 3.5]. It is apparent that the cosine transform (1.7) is a special case of (1.2) for $K=I$ from (1.3).

The right-hand side of (1.7) is the support function of a convex body in $\mathbb{R}^{n}$, called the zonoid of $\mu$, see [13, Sec. 3.5]. Similarly, $\mathbf{E}|\langle\xi, u\rangle|$ is the support function of the zonoid of $\xi$, see [15] and [10]. Note that

$$
\mathbf{E}|\langle\xi, u\rangle|=\mathbf{E} h(u I, \xi)=\mathbf{E} h(\xi I, u), \quad u \in \mathbb{R}^{n} .
$$

Two integrable random vectors $\xi$ and $\eta$ satisfying (1.6) are said to be zonoid equivalent, see, e.g., [10]. The extent to which zonoid equivalent random vectors can have different distributions is explored in [9].

In the following we replace the function $|\langle u, x\rangle|$ above with $h(u K, x)$ with the aim to describe convex bodies $K$ such that the equality

$$
\mathbf{E} h(u K, \xi)=\mathbf{E} h(u K, \eta), \quad u \in \mathbb{R}^{n}
$$

yields the zonoid equivalence of $\xi$ and $\eta$, hence, also (1.5) for all one-homogeneous even $f$. The zonoid equivalence of $\xi$ and $\eta$ immediately yields (1.8). The inverse implication is not true for general $K$, as the following example shows. 
Example 1.2. Let $K=[-1,1]^{2}$ be the $\ell_{\infty}$-ball in $\mathbb{R}^{2}$. For integrable $\xi=\left(\xi_{1}, \xi_{2}\right)$, we have

$$
\mathbf{E} h(u K, \xi)=\mathbf{E}\left|u_{1} \xi_{1}\right|+\mathbf{E}\left|u_{2} \xi_{2}\right|,
$$

which is determined by the first absolute moments of the coordinates of $\xi$, and so carries considerably less information than the zonoid of $\xi$.

Example 1.3. Let $K=B_{2}$ be the unit Euclidean ball in $\mathbb{R}^{n}$. Then

$$
\mathbf{E} h\left(u B_{2}, \xi\right)=\mathbf{E}\left(u_{1}^{2} \xi_{1}^{2}+\cdots+u_{n}^{2} \xi_{n}^{2}\right)^{1 / 2}
$$

is the expected Euclidean norm of $u \xi$. Such expectations carry the same information as the zonoid of $\xi$ if all components of $\xi$ are non-negative, see Theorems 3.2 and 4.15 .

Note that $\mathbf{E} h(\xi K, u)$ is the support function of a convex body $\mathbf{E}(\xi K)$ called the expectation of $\xi K$, see [7]. In particular, the zonoid of $\xi$ is the expectation of the random segment $\xi I$, see [15].

It is possible to define an $\mathrm{L}^{p}$-version of zonoid equivalence using the $p$ th moments of $|\langle u, \xi\rangle|$ or their max-combinations. In this respect, the following result is important.

Theorem 1.4 (see [16, Th. 1.1]). Let $\xi$ and $\eta$ be $\alpha$-integrable random vectors in $\mathbb{R}_{+}^{n}$ with $\alpha \in(0,2)$. Then

$$
\mathbf{E}|\langle u, \xi\rangle|^{\alpha}=\mathbf{E}|\langle u, \eta\rangle|^{\alpha}, \quad u \in \mathbb{R}^{n}
$$

if and only if

$$
\mathbf{E}\left(\max _{i=1, \ldots, n} u_{i} \xi_{i}\right)^{\alpha}=\mathbf{E}\left(\max _{i=1, \ldots, n} u_{i} \eta_{i}\right)^{\alpha}, \quad u \in \mathbb{R}_{+}^{n} .
$$

\subsection{Structure of the paper}

The main aim of this paper is to study the diagonal Minkowski class generated by a symmetric convex body $K$. In Section 2 , an argument, relating injectivity and surjectivity of a linear map, establishes the equivalence of the injectivity of the $K$-transform (1.2) and the fact that the (signed) linear combinations of support functions of diagonal bodies are dense in the family of support functions of all symmetric convex bodies.

In Section 3 we explore in which cases $T_{K}$ is injective assuming that $K$ is a generalised zonoid. The key argument relies on deriving that the identity $\mathbf{E} f(\xi \zeta)=\mathbf{E} f(\eta \zeta)$ for integrable symmetric random vectors $\xi, \eta$, an independent random vector $\zeta$, and all onehomogeneous even functions $f$ yields that $\mathbf{E} f(|\xi|)=\mathbf{E} f(|\eta|)$, where $|\xi|$ and $|\eta|$ are vectors composed of the absolute values of the components of $\xi$ and $\eta$, respectively. In dimensions two and more it is generally not possible to take $\zeta$ out of $f(\xi \zeta)$, and the proof goes via letting $f$ be the product of powers of all arguments.

In order to show that (1.8) implies (1.6) (hence, (1.5)) for all integrable symmetric random vectors one has to assume that $K$ does not possess certain symmetries. In particular, it is necessary that $K$ is not symmetric with respect to any coordinate hyperplane. The major result of the paper, Theorem 3.6, shows that (1.8) implies (1.6) if and only if $K$ has singe-point support sets in directions of all coordinate axes and satisfies a specific asymmetry condition which is stronger than $K$ not being unconditional.

Section 3.3 relates the $K$-transform of surface area measures to the mixed volumes $V(L, \ldots, L, u K)$ involving the diagonally transformed $K$ and discusses the corresponding uniqueness problem for the convex body $L$. 
Finally, in Section 4 it is shown that diagonally transformed $\ell_{p}$-balls are naturally related to distributions of one-sided strictly stable random vectors. That section complements the study of the geometric interpretation of stable laws, initiated in [6] for the symmetric setting. This connection is exploited to show that $\ell_{p}$-balls (which are not necessarily generalised zonoids) are unconditionally universal. Some further properties of convex bodies obtained as diagonal transforms of $\ell_{p}$-balls are derived.

The Appendix contains a result that extends the setup of Section 2 to more general families of transformations.

\section{Injectivity of the $K$-transform}

Convex bodies that can be approximated in the Hausdorff metric by positive linear combinations of a convex body $K$ and its rotations form a Minkowski class $\mathscr{M}$; its members are called $\mathscr{M}$-bodies, see [14]. Furthermore, $M$ is a generalised $\mathscr{M}$-body if

$$
M+L_{1}=L_{2}
$$

with $L_{1}, L_{2}$ being $\mathscr{M}$-bodies.

Denote by $\mathscr{K}_{0}$ the family of origin symmetric convex bodies. A convex body $K \in \mathscr{K}_{0}$ is called centrally universal if the expansion of its support function into spherical harmonics contains non-zero harmonics of all even orders, see [12]. It is shown in [14, Th. 2] that this holds if and only if the family of generalised $\mathscr{M}$-bodies is dense in $\mathscr{K}_{0}$. In particular, if $K$ is a symmetric segment, the corresponding $K$-bodies are zonoids and generalised zonoids are dense in $\mathscr{K}_{0}$.

In this paper, we consider a similar situation, but instead of rotations we apply to $K$ diagonal transformations. Recall that the rotations/scalings and diagonal transformations are equivalent if $K$ is the segment $I$ given by (1.3).

Theorem 2.1. Let $K \in \mathscr{K}_{0}$. The linear combinations of the support functions of diagonal bodies generated by $K$ are dense in the family of continuous even functions on the unit sphere with the uniform metric (hence, in the family of support functions of symmetric convex bodies) if and only if the $K$-transform is injective on finite even signed measures on $\mathbb{S}^{n-1}$.

Proof. Necessity. The support function of the segment $I$ can be approximated by linear combinations of support functions of diagonal bodies, and it suffices to refer to the injectivity property of the cosine transform.

Sufficiency. Denote by $\mathscr{M}_{e}$ the family of finite signed even measures on the unit sphere and by $C_{e}$ the family of even continuous functions on the unit sphere. We will prove that the image of $T_{K}$ restricted to measures with densities from $C_{e}$ is dense in $C_{e}$. Note that $T_{K}$ is continuous in the uniform metric on $C_{e}$. Its adjoint operator $T_{K}^{\prime}$ acts on $\mathscr{M}_{e}$, so that

$$
\int_{\mathbb{S}^{n-1}} f d\left(T_{K}^{\prime} \nu\right)=\int_{\mathbb{S}^{n-1}}\left(T_{K} f\right) d \nu, \quad f \in C_{e} .
$$

Assume that $T_{K}^{\prime} \nu=0$, that is, $\nu$ belongs to the kernel of $T_{K}^{\prime}$. Then

$$
\int_{\mathbb{S}^{n-1}} \int_{\mathbb{S}^{n-1}} h(u K, v) f(u) d u \nu(d v)=0
$$


for all $f \in C_{e}$, whence

$$
\int_{\mathbb{S}^{n-1}} h(v K, u) \nu(d v)=0, \quad u \in \mathbb{S}^{n-1}
$$

By the injectivity assumption, $\nu=0$. The triviality of the kernel of $T_{K}^{\prime}$ yields that the range of $T_{K}$ is dense in $C_{e}$, see [17, Th. III.4.5].

In analogy with [14], $K$ satisfying one of the equivalent conditions in Theorem 2.1 is called diagonally universal or D-universal.

Proposition 2.2. A set $K \in \mathscr{K}_{0}$ is D-universal if and only if (1.8) for any two symmetric integrable random vectors $\xi$ and $\eta$ implies their zonoid equivalence.

Proof. Necessity. A symmetric integrable random vector $\xi$ yields an even finite measure on $\mathbb{S}^{n-1}$ given by

$$
\mu_{\xi}(A)=\mathbf{E}\left(\|\xi\| \mathbb{1}_{\xi /\|\xi\| \in A}\right)
$$

for all Borel $A \subset \mathbb{S}^{n-1}$, where $\|x\|$ denotes the Euclidean norm of $x \in \mathbb{R}^{n}$. Then

$$
\mathbf{E} h(u K, \xi)=\int_{\mathbb{S}^{n-1}} h(u K, v) \mu_{\xi}(d v), \quad u \in \mathbb{R}^{n} .
$$

Thus, if $T_{K}$ is injective, then (1.8) yields $\mu_{\xi}=\mu_{\eta}$, whence (1.6) holds and $\xi$ and $\eta$ are zonoid equivalent.

Sufficiency. A finite measure $\mu$ on $\mathbb{S}^{n-1}$ yields an integrable random vector $\xi=$ $\mu\left(\mathbb{S}^{n-1}\right) \xi^{\prime}$, where $\xi^{\prime}$ is distributed according to the normalised $\mu$. Then

$$
\int_{\mathbb{S}^{n-1}} h(u K, v) \mu(d v)=\mathbf{E} h(u K, \xi), \quad u \in \mathbb{R}^{n} .
$$

Let $\eta$ be generated by $\nu$ in the same way. If $T_{K} \mu=T_{K} \nu$, then $\xi$ and $\eta$ are zonoid equivalent, whence $\mu=\nu$ by the injectivity of the standard cosine transform. The injectivity of $T_{K}$ on signed even measures follows from the Jordan decomposition into their positive and negative parts.

A convex body is called unconditional if it is symmetric with respect to all coordinate hyperplanes $\left\{x=\left(x_{1}, \ldots, x_{n}\right) \in \mathbb{R}^{n}: x_{i}=0\right\}, i=1, \ldots, n$; the family of unconditional bodies is denoted by $\mathscr{K}_{s}$.

Theorem 2.3. Let $K \in \mathscr{K}_{s}$. The linear combinations of support functions of diagonal bodies generated by $K$ are dense in the family of support functions of unconditional convex bodies if and only if (1.8) for all $u \in \mathbb{R}^{n}$ and any two integrable random vectors $\xi$ and $\eta$ in $\mathbb{R}^{n}$ implies the zonoid equivalence of $|\xi|=\left(\left|\xi_{1}\right|, \ldots,\left|\xi_{n}\right|\right)$ and $|\eta|=\left(\left|\eta_{1}\right|, \ldots,\left|\eta_{n}\right|\right)$.

Proof. Since $K$ is unconditional, (1.8) holds if and only if

$$
\mathbf{E} h(u K,|\xi|)=\mathbf{E} h(u K,|\eta|),
$$

whence it suffices to assume that $\xi$ and $\eta$ take values in $\mathbb{R}_{+}^{n}$ and let $u \in \mathbb{R}_{+}^{n}$. 
Necessity. By assumption, the support function of the $\ell_{1}$-ball $B_{1}$ can be approximated by linear combinations of support functions of diagonal bodies, so that

$$
\mathbf{E} \max \left(u_{1} \xi_{1}, \ldots, u_{n} \xi_{n}\right)=\mathbf{E} \max \left(u_{1} \eta_{1}, \ldots, u_{n} \eta_{n}\right)
$$

for all $u=\left(u_{1}, \ldots, u_{n}\right) \in \mathbb{R}_{+}^{n}$. By Theorem 1.4, $\xi$ and $\eta$ are zonoid equivalent.

Sufficiency. The proof replicates the proof of sufficiency in Theorem 2.1 by restricting measures and functions onto the unit sphere intersected with $\mathbb{R}_{+}^{n}$.

The property of $K$ (not necessarily belonging to $\mathscr{K}_{s}$ ) formulated in Theorem 2.3 can be interpreted as the injectivity of the $K$-transform over unconditional measures. In this case $K$ is called unconditionally D-universal.

Example 2.4. It follows from Theorem 1.4 that $B_{1}$ is unconditionally D-universal. The derivation of similar results for $B_{q}$ with $q>1$ requires the methods of Section 4 ,

The following result shows that a scaled $\ell_{\infty}$-ball is allowed as a summand of a $D$ universal $K$ if the rest is D-universal.

Proposition 2.5. Let $B_{\infty}=\left\{x \in \mathbb{R}^{n}:\left|x_{i}\right| \leq 1, i=1, \ldots, n\right\}$ be the $\ell_{\infty}$-ball in $\mathbb{R}^{n}$, and let $K=K_{0}+w B_{\infty}$ for $w \in \mathbb{R}_{+}^{n}$ and $K_{0} \in \mathscr{K}_{0}$. Then (1.8) implies

$$
\mathbf{E} h\left(u K_{0}, \xi\right)=\mathbf{E} h\left(u K_{0}, \eta\right), \quad u \in \mathbb{R}^{n} .
$$

Proof. Since $w B_{\infty}$ equals the sum of $w_{i}\left[-e_{i}, e_{i}\right], i=1, \ldots, n$, it is possible to proceed by induction. Let $K=K_{0}+\left[-e_{1}, e_{1}\right]$. If $u=e_{1}$, then

$$
\mathbf{E} h(u K, \xi)=\left(h\left(K_{0}, e_{1}\right)+1\right) \mathbf{E}\left|\xi_{1}\right| .
$$

Thus, (1.8) implies $\mathbf{E}\left|\xi_{1}\right|=\mathbf{E}\left|\eta_{1}\right|$. For $u \in \mathbb{R}^{n}$,

$$
\mathbf{E} h(u K, \xi)=\mathbf{E} h\left(u K_{0}, \xi\right)+\left|u_{1}\right| \mathbf{E}\left|\xi_{1}\right|,
$$

whence (2.2) holds.

Thus, if $K_{0}$ is (unconditionally) D-universal, then also the convex body $K_{0}+v B_{\infty}$ is (unconditionally) D-universal for all $v \in \mathbb{R}^{n}$.

Remark 2.6. In order to eliminate the dependence of the diagonal transformations on the chosen coordinate system, it is possible to incorporate a single rotation of $K$, that is, consider the Minkowski class generated by $c_{1} u_{1} O K+\cdots c_{m} u_{m} O K$, where $O$ is an orthogonal matrix 11 The universality holds if and only if a rotation of $K$ is D-universal.

Remark 2.7. If $p \in[1, \infty)$, the $L_{p}$-variant of the $T_{K}$-transform is defined by letting

$$
T_{K}^{p} \mu=\left(\int_{\mathbb{S}^{n-1}} h(u K, v)^{p} \mu(d v)\right)^{1 / p}
$$

equivalently, as $\left(\mathbf{E} h(u K, \xi)^{p}\right)^{1 / p}$ for a $p$-integrable random vector $\xi$. Furthermore, it is possible to consider radial sums of diagonally transformed convex bodies or add them in the Blaschke sense.

\footnotetext{
${ }^{1}$ The authors are grateful to Daniel Hug for suggesting this construction.
} 


\section{Diagonal universality of generalised zonoids}

\subsection{Unconditional universality}

For $u \neq 0$, let

$$
F(K, u)=\{x \in K:\langle x, u\rangle=h(K, u)\}
$$

denote the support set of $K$ in direction $u$.

Lemma 3.1. For a generalised zonoid $K$, the support sets $F\left(K, e_{i}\right)$ in directions of the standard basis vectors $e_{1}, \ldots, e_{n}$ are all singletons if and only if the representing measure of $K$ vanishes on $S_{0}=\left\{v \in \mathbb{S}^{n-1}: v_{1} \cdots v_{n}=0\right\}$.

Proof. By [13, Lemma 3.5.6], the support set $F(K, u)$ is a singleton for $u \neq 0$ if and only if the representing measure vanishes on $S_{u}=\left\{x \in \mathbb{S}^{n-1}:\langle x, u\rangle=0\right\}$. Now the claim follows from the fact that $S_{0}=\cup_{i} S_{e_{i}}$.

Recall that $K$ is unconditionally D-universal if (1.8) implies that $\left(\left|\xi_{1}\right|, \ldots,\left|\xi_{n}\right|\right)$ and $\left(\left|\eta_{1}\right|, \ldots,\left|\eta_{n}\right|\right)$ are zonoid equivalent.

Theorem 3.2. Let $K$ be a generalised zonoid such that all support sets $F\left(K, e_{i}\right), i=$ $1, \ldots, n$, are singletons. Then $K$ is unconditionally D-universal.

The proof of Theorem 3.2 is based on the following two lemmas. For $x \in \mathbb{R}^{n}$ and $\alpha \in \mathbb{R}^{n}$, write

$$
[x]^{\alpha}=\prod_{i=1}^{n}\left|x_{i}\right|^{\alpha_{i}},
$$

and

$$
\begin{aligned}
|x| & =\left(\left|x_{1}\right|, \ldots,\left|x_{n}\right|\right), \\
\operatorname{sign}(x) & =\left(\operatorname{sign}\left(x_{1}\right), \ldots, \operatorname{sign}\left(x_{n}\right)\right)
\end{aligned}
$$

for the componentwise absolute values and signs of $x \in \mathbb{R}^{n}$. We use the convention $0^{0}=1$. For $E \subseteq\{1, \ldots, n\}$, denote

$$
A_{E}=\left\{x \in \mathbb{R}^{n}: x_{i} \neq 0, i \in E, x_{j}=0, j \notin E\right\},
$$

and let

$$
\Delta_{E}=\left\{\alpha \in \mathbb{R}_{+}^{n}: \sum \alpha_{i}=1, \alpha_{j}=0, j \notin E\right\}
$$

be the unit simplex in the linear subspace generated by $A_{E}$. Let $A_{\varnothing}=\{0\}$ and $\Delta_{\varnothing}=\{0\}$.

Lemma 3.3. Let $\xi$ and $\eta$ be two random vectors in $(0, \infty)^{n}$. If

$$
\mathbf{E}[\xi]^{\alpha}=\mathbf{E}[\eta]^{\alpha}<\infty
$$

for all $\alpha$ from an open set in $\mathbb{R}^{n}$, then $\xi$ and $\eta$ are identically distributed.

Proof. By passing to componentwise $\operatorname{logarithms} \tilde{\xi}=\log \xi$ and $\tilde{\eta}=\log \eta$, we see that the Laplace transforms of $\tilde{\xi}$ and $\tilde{\eta}$ agree on an open set, and the result follows from [3, Lemma 7]. 
Lemma 3.4. Let $\xi$ and $\eta$ be integrable random vectors in $\mathbb{R}_{+}^{n}$. Then $\xi$ and $\eta$ are zonoid equivalent if and only if

$$
\mathbf{E}\left([\xi]^{\alpha} \mathbb{1}_{\xi \in A_{E}}\right)=\mathbf{E}\left([\eta]^{\alpha} \mathbb{1}_{\eta \in A_{E}}\right)
$$

for all non-empty $E \subseteq\{1, \ldots, n\}$ and $\alpha$ from a relatively open subset of the unit simplex $\Delta_{E}$.

Proof. Necessity follows from the fact that, for all $\alpha \in \Delta_{E}$, the expectations in (3.2) are taken of a one-homogenous even function of $\xi$ and $\eta$, so that Theorem 1.1 applies.

Sufficiency. Fix $E \subseteq\{1, \ldots, n\}$ of cardinality at least 2, and assume that (3.2) holds for $\alpha$ from a relatively open neighbourhood of some $\beta \in \Delta_{E}$. Then

$$
c=\mathbf{E}\left([\xi]^{\beta} \mathbb{1}_{\xi \in A_{E}}\right)=\mathbf{E}\left([\eta]^{\beta} \mathbb{1}_{\eta \in A_{E}}\right) .
$$

Assume that $c>0$ and define probability measures $\mathbf{Q}$ and $\mathbf{Q}^{*}$ with densities

$$
\frac{d \mathbf{Q}}{d \mathbf{P}}=\frac{1}{c}[\xi]^{\beta} \mathbb{1}_{\xi \in A_{E}}, \quad \frac{d \mathbf{Q}^{*}}{d \mathbf{P}}=\frac{1}{c}[\eta]^{\beta} \mathbb{1}_{\eta \in A_{E}},
$$

and let $\mathbf{E}_{\mathbf{Q}}$ and $\mathbf{E}_{\mathbf{Q}^{*}}$ denote the expectations with respect to $\mathbf{Q}$ and $\mathbf{Q}^{*}$, respectively.

Then (3.2) implies

$$
\mathbf{E}_{\mathbf{Q}} \prod_{i \in E} \xi_{i}^{\gamma_{i}}=\mathbf{E}_{\mathbf{Q}^{*}} \prod_{i \in E} \eta_{i}^{\gamma_{i}}
$$

for all $\gamma$ from a neighbourhood of the origin and such that $\sum \gamma_{i}=0$ and $\gamma_{j}=0$ for $j \notin E$. Fix any $k \in E$ and notice that $\gamma_{k}=-\sum_{i \neq k} \gamma_{i}$. Therefore,

$$
\mathbf{E}_{\mathbf{Q}} \prod_{i \in E, i \neq k}\left(\frac{\xi_{i}}{\xi_{k}}\right)^{\gamma_{i}}=\mathbf{E}_{\mathbf{Q}^{*}} \prod_{i \in E, i \neq k}\left(\frac{\eta_{i}}{\eta_{k}}\right)^{\gamma_{i}}
$$

for $\gamma_{i}, i \neq k$, from an open set in the subspace of $\mathbb{R}^{n}$ generated by the basis vectors $e_{i}$, $i \in E \backslash\{k\}$.

By Lemma 3.3 applied to this subspace of $\mathbb{R}^{n}$, the distributions of $\xi_{i} / \xi_{k}, i \in E \backslash\{k\}$, under $\mathbf{Q}$ and $\eta_{i} / \eta_{k}, i \in E \backslash\{k\}$, under $\mathbf{Q}^{*}$ coincide. These vectors can be extended by placing 1 at the component number $k$ and 0 at all components outside $E$. Therefore,

$$
\begin{aligned}
\mathbf{E}\left(|\langle u, \xi\rangle| \mathbb{1}_{\xi \in A_{E}}\right) & =c \mathbf{E}_{\mathbf{Q}}\left(\left|\left\langle u, \xi_{k}^{-1} \xi\right\rangle\right|\left[\xi_{k}^{-1} \xi\right]^{-\beta} \mathbb{1}_{\xi \in A_{E}}\right) \\
& =c \mathbf{E}_{\mathbf{Q}^{*}}\left(\left|\left\langle u, \eta_{k}^{-1} \eta\right\rangle\right|\left[\eta_{k}^{-1} \eta\right]^{-\beta} \mathbb{1}_{\eta \in A_{E}}\right) \\
& =\mathbf{E}\left(|\langle u, \eta\rangle| \mathbb{1}_{\eta \in A_{E}}\right) .
\end{aligned}
$$

This also holds if $c=0$ in (3.3), and for $E=\varnothing$. If $E$ is a singleton, then this holds by (3.2). Taking the sum over all $E \subseteq\{1, \ldots, n\}$ shows that $\xi$ and $\eta$ are zonoid equivalent.

Proof of Theorem 3.2. It follows from (1.8) and (1.4) that

$$
\int_{\mathbb{S}^{n-1}} \mathbf{E}|\langle u, \xi v\rangle| \nu(d v)=\int_{\mathbb{S}^{n-1}} \mathbf{E}|\langle u, \eta v\rangle| \nu(d v), \quad u \in \mathbb{R}^{n}
$$

By splitting $\nu$ into the positive and negative parts and referring to the equivalence of (1.6) and (1.5) (see [9, Th. 2]), it is possible to conclude that

$$
\int_{\mathbb{S}^{n-1}} \mathbf{E} f(\xi v) \nu(d v)=\int_{\mathbb{S}^{n-1}} \mathbf{E} f(\eta v) \nu(d v)
$$


for all one-homogeneous even functions $f$.

Taking $f(x)=[x]^{\alpha} \mathbb{1}_{x \in A_{E}}$ for non-empty $E \subseteq\{1, \ldots, n\}$ yields that

$$
\int_{\mathbb{S}^{n-1}} \mathbf{E}\left([\xi v]^{\alpha} \mathbb{1}_{\xi v \in A_{E}}\right) \nu(d v)=\int_{\mathbb{S}^{n-1}} \mathbf{E}\left([\eta v]^{\alpha} \mathbb{1}_{\eta v \in A_{E}}\right) \nu(d v), \quad \alpha \in \Delta_{E}
$$

Taking into account that $\nu$ vanishes on the set $\left\{x \in \mathbb{S}^{n-1}: x_{1} \cdots x_{n}=0\right\}$ by Lemma 3.1, we may assume that none of the components of $v$ from the integration domain vanishes, whence

$$
\int_{\mathbb{S}^{n-1}}[v]^{\alpha} \nu(d v) \mathbf{E}\left([\xi]^{\alpha} \mathbb{1}_{\xi \in A_{E}}\right)=\int_{\mathbb{S}^{n-1}}[v]^{\alpha} \nu(d v) \mathbf{E}\left([\eta]^{\alpha} \mathbb{1}_{\eta \in A_{E}}\right), \quad \alpha \in \Delta_{E} .
$$

The integral of $[v]^{\alpha}$ does not vanish for $\alpha$ being any basis vector. Indeed, if it vanishes for $\alpha=e_{i}$, then

$$
0=\int_{\mathbb{S}^{n-1}}[v]^{e_{i}} \nu(d v)=\int_{\mathbb{S}^{n-1}}\left|\left\langle v, e_{i}\right\rangle\right| \nu(d v)=h\left(K, e_{i}\right),
$$

whence $K$ is a subset of a coordinate hyperplane.

Hence,

$$
\mathbf{E}\left([\xi]^{\alpha} \mathbb{1}_{\xi \in A_{E}}\right)=\mathbf{E}\left([\eta]^{\alpha} \mathbb{1}_{\eta \in A_{E}}\right)
$$

for all $\alpha$ from a neighbourhood in $\Delta_{E}$ of any basis vector $e_{k}$ with $k \in E$. By Lemma 3.4, $|\xi|$ and $|\eta|$ are zonoid equivalent.

Corollary 3.5. Each symmetric convex body in $\mathbb{R}^{2}$ is either a diagonal transform of $B_{\infty}$ or it is unconditionally D-universal.

Proof. Recall that in $\mathbb{R}^{2}$ each symmetric convex body $K$ is a zonoid, say with representing measure $\nu$ on $S^{1}$. Restricting $\nu$ to $S^{1} \backslash\left\{ \pm e_{1}, \pm e_{2}\right\}$ and to $\left\{ \pm e_{1}, \pm e_{2}\right\}$ yields a decomposition $K=K_{0}+w B_{\infty}$ where $K_{0}$ is a zonoid with support sets $F\left(K_{0}, e_{i}\right), i=1,2$, being singletons and $w \in \mathbb{R}^{2}$. If $K_{0}$ is non-trivial, then it is unconditionally D-universal by Theorem 3.2, and thus $K$ is unconditionally D-universal by Proposititon 2.5 ,

Notice that the generalisation of Corollary 3.5 to dimension $n \geq 3$ fails, even for zonoids, because the restriction of $\nu$ to $\mathbb{S}^{n-1}$ and $\mathbb{S}^{n-1} \backslash S_{0}$ does not lead to a decomposition of the form considered in Proposition 2.5.

\subsection{Diagonal universality}

Theorem 3.2 yields that all generalised zonoids with single-point support sets in directions of all basis vectors are unconditionally D-universal. To obtain the D-universality property, one has to be able to recover the distribution of signs of the components of $\xi$ from $\mathrm{E} h(u K, \xi)$. For $J \subset\{1, \ldots, n\}$ and $\alpha \in \mathbb{R}_{+}^{n}$, introduce the function

$$
f_{\alpha, J}(x)=[x]^{\alpha} \prod_{i \in J} \operatorname{sign}\left(x_{i}\right), \quad x \in \mathbb{R}^{n} .
$$

Denoting by $t^{\langle\beta\rangle}=|t|^{\beta} \operatorname{sign}(t)$ the signed $\beta$-power of $t \in \mathbb{R}$ for $\beta \geq 0$, it is possible to write

$$
f_{\alpha, J}(x)=\prod_{i \in J} x_{i}^{\left\langle\alpha_{i}\right\rangle} \prod_{i \notin J}\left|x_{i}\right|^{\alpha_{i}} .
$$


For a symmetric convex body $K$ in $\mathbb{R}^{n}$ and $J \subseteq\{1, \ldots, n\}$ the projection of $K$ on the subspace $\mathbb{R}^{J}$ spanned by $\left\{e_{j}, j \in J\right\}$ is denoted by $K_{J}$. Moreover, denote $\mathcal{S}^{n}=\{-1,1\}^{n}$ and $\mathcal{S}^{J}=\{-1,1\}^{J}$, and write $\sigma(t)=\prod_{j \in J} \operatorname{sign}\left(t_{j}\right)$ for $t \in \mathbb{R}^{J}$ or $t \in \mathcal{S}^{J}$. For $s \in \mathcal{S}^{n}$, denote by $R_{s}$ the set of all $x \in \mathbb{R}^{n}$ such that $\operatorname{sign}(x)=s$.

A symmetric convex body $L$ in $\mathbb{R}^{J}$ is said to satisfy the asymmetry condition if

$$
\sum_{s \in \mathcal{S}^{J}, \sigma(s)=1} s L \neq \sum_{s \in \mathcal{S}^{J}, \sigma(s)=-1} s L .
$$

For this, the cardinality of $J$ should be even; (AS) never holds if the cardinality of $J$ is odd. It is easy to see that (ASS) is equivalent to $L$ being not unconditional if the cardinality of $J$ is 2 ; in higher dimensions, (AS) is stronger than $L$ not being unconditional as can be seen from Example 3.10 below.

Theorem 3.6. Let $K$ be a generalised zonoid in $\mathbb{R}^{n}$ such that all support sets $F\left(K, e_{i}\right), i=$ $1, \ldots, n$, are singletons. Then $K$ is D-universal if and only if $K_{J}$ satisfies the asymmetry condition (AS) for each non-empty $J \subseteq\{1, \ldots, n\}$ of even cardinality.

To prove sufficiency we need a generalisation of Lemma 3.4 .

Lemma 3.7. Two symmetric integrable random vectors $\xi$ and $\eta$ in $\mathbb{R}^{n}$ are zonoid equivalent if and only if

$$
\mathbf{E}\left(f_{\alpha, J}(\xi) \mathbb{1}_{\xi \in A_{E}}\right)=\mathbf{E}\left(f_{\alpha, J}(\eta) \mathbb{1}_{\eta \in A_{E}}\right)
$$

for all $J \subseteq E \subseteq\{1, \ldots, n\}$ with $E \neq \varnothing$ and all $\alpha$ from a relatively open set in $\Delta_{E}$.

Proof. Necessity. Theorem 1.1 implies (3.5) if the cardinality of $J$ is even, otherwise both sides of (3.5) vanish.

Sufficiency. Let $s \in\{-1,0,1\}^{n}$ and $E$ be such that $s_{i} \neq 0$ for $i \in E$ and $s_{i}=0$ for $i \notin E$. Then

$$
\sum_{J \subseteq E} \prod_{i \in J} s_{i} \operatorname{sign}\left(x_{i}\right) \mathbb{1}_{x \in A_{E}}=2^{m} \mathbb{1}_{\operatorname{sign}(x)=s}, \quad x \in \mathbb{R}^{n},
$$

where $m$ is the cardinality of $E$. Using this in (3.5), we have

$$
\mathbf{E}\left([\xi]^{\alpha} \mathbb{1}_{\xi \in A_{E}} \mathbb{1}_{\operatorname{sign}(\xi)=s}\right)=\mathbf{E}\left([\eta]^{\alpha} \mathbb{1}_{\eta \in A_{E}} \mathbb{1}_{\operatorname{sign}(\eta)=s}\right) .
$$

By Lemma 3.4, $|\xi| \mathbb{1}_{\operatorname{sign}(\xi)=s}$ and $|\eta| \mathbb{1}_{\operatorname{sign}(\eta)=s}$ are zonoid equivalent, whence

$$
\mathbf{E}\left(|\langle u,|\xi|\rangle| \mathbb{1}_{\operatorname{sign}(\xi)=s}\right)=\mathbf{E}\left(|\langle u,|\eta|\rangle| \mathbb{1}_{\operatorname{sign}(\eta)=s}\right), \quad u \in \mathbb{R}^{n} .
$$

Since this equality holds for all $u$, it is possible to replace $u$ by $u s$, and it remains to take the sum over $s$.

Proof of Theorem 3.6. Necessity. Assume that $K_{J}$ does not satisfy (AS) for some nonempty $J$ with even cardinality. Then

$$
\sum_{s \in \mathcal{S}^{J}, \sigma(s)=1} s L=\sum_{s \in \mathcal{S}^{J}, \sigma(s)=-1} s L
$$

holds for all convex bodies $L$ in $\mathbb{R}^{J}$, whose support functions lie in the closed linear hull of support functions of the diagonal transforms of $K_{J}$. Since the unit segment $I$ in $\mathbb{R}^{J}$ 
does not satisfy (3.6), I cannot be approximated which is a contradiction to $K$ being D-universal.

Sufficiency. The representing measure of $K$ is denoted by $\nu$. Let $\xi$ and $\eta$ be symmetric random vectors. Repeating the first step in the proof of Theorem 3.2, we arrive at

$$
\int_{\mathbb{S}^{n-1}} \mathbf{E}\left(f_{\alpha, J}(\xi v) \mathbb{1}_{\xi v \in A_{E}}\right) \nu(d v)=\int_{\mathbb{S}^{n-1}} \mathbf{E}\left(f_{\alpha, J}(\eta v) \mathbb{1}_{\eta v \in A_{E}}\right) \nu(d v)
$$

for all $\alpha \in \Delta_{E}$ and $J \subseteq E \subseteq\{1, \ldots, n\}$ where $J \neq \varnothing$ and $J$ has even cardinality. As $\nu$ does not charge $S_{0}$,

$$
\int_{\mathbb{S}^{n-1}} f_{\alpha, J}(v) \nu(d v)\left(\mathbf{E}\left(f_{\alpha, J}(\xi) \mathbb{1}_{\xi \in A_{E}}\right)-\mathbf{E}\left(f_{\alpha, J}(\eta) \mathbb{1}_{\eta \in A_{E}}\right)\right)=0 .
$$

Now fix $J$. We show that

$$
\int_{\mathbb{S}^{n-1}} f_{\alpha, J}(v) \nu(d v) \neq 0
$$

for some $\alpha \in \Delta_{J} \subseteq \Delta_{E}$, and therefore also for all $\alpha$ in a relatively open set in $\Delta_{E}$ by continuity of $[x]^{\alpha}$ in $\alpha$ for $x$ with non-vanishing components. Assume that

$$
\int_{\mathbb{S}^{J}} f_{\alpha, J}(w) \nu_{J}(d w)=\int_{\mathbb{S}^{n-1}} f_{\alpha, J}(v) \nu(d v)=0, \quad \alpha \in \Delta_{J}
$$

where $\mathbb{S}^{J}$ is the unit sphere in $\mathbb{R}^{J}$ and $\nu_{J}$ is the representing measure of $K_{J}$. We assume that $J=\{1, \ldots, n\}$ with an even $n$ and $\nu=\nu_{J}$, the general case being similar. Then

$$
\begin{aligned}
\int_{\mathbb{S}^{n-1}} f_{\alpha, J}(v) \nu(d v) & =\int_{\mathbb{S}^{n-1}} \prod_{i=1}^{n} v_{i}^{\left\langle\alpha_{i}\right\rangle} \nu(d v) \\
& =\sum_{s \in \mathcal{S}^{n}} \int_{\mathbb{S}^{n-1} \cap R_{s}}[v]^{\alpha} \sigma(v) \nu(d v) \\
& =\sum_{s \in \mathcal{S}^{n}} \int_{\mathbb{S}^{n-1} \cap R_{s}}[v]^{\alpha} \sigma(s) \nu(d v),
\end{aligned}
$$

where the last equality holds because $\sigma(v)=\sigma(s)$ for $v \in R_{s}$. Since the expression in the last line is zero for $\alpha \in \Delta_{J}$, Lemma 3.4 implies that

$$
0=\sum_{s \in \mathcal{S}^{n}} \sigma(s) \int_{\mathbb{S}^{n-1} \cap R_{s}}|\langle u,|v|\rangle| \nu(d v)=\sum_{s \in \mathcal{S}^{n}} \sigma(s) \int_{\mathbb{S}^{n-1} \cap R_{s}}|\langle s u, v\rangle| \nu(d v), \quad u \in \mathbb{R}^{n} .
$$

Multiplying this with $\sigma(t)$, replacing $u$ with $u$, taking the sum over $t \in \mathcal{S}^{n}$, and writing $t$ instead of $t s$ yield that

$$
0=\sum_{t \in \mathcal{S}^{n}} \sigma(t) \sum_{s \in \mathcal{S}^{n}} \int_{\mathbb{S}^{n-1} \cap R_{s}}|\langle t u, v\rangle| \nu(d v)=\sum_{t \in \mathcal{S}^{n}} \sigma(t) \int_{\mathbb{S}^{n-1}}|\langle t u, v\rangle| \nu(d v), \quad u \in \mathbb{R}^{n} .
$$

Therefore, for $u \in \mathbb{R}^{n}$

$$
\sum_{s \in \mathcal{S}^{n}} \sigma(s) h(K, s u)=\sum_{s \in \mathcal{S}^{n}} \sigma(s) \int_{\mathbb{S}^{n-1}}|\langle v, s u\rangle| \nu(d v)=0 .
$$


This contradicts (AS), so (3.7) is proven.

If the cardinality of $J$ is odd, then the symmetry of $\xi$ and $\eta$ yields that (3.5) holds with both sides vanishing. If $J=\varnothing$ and $E \neq \varnothing$, then (3.5) holds since $|\xi|$ and $|\eta|$ are zonoid equivalent by Theorem 3.2. Finally, the result follows from Lemma 3.7.

Corollary 3.8. A symmetric convex body $K$ in $\mathbb{R}^{2}$ is D-universal if and only if $K$ is not unconditional.

Proof. Decompose $K$ as in the proof of Corollary 3.5. If $K$ is not unconditional, then $K_{0}$ is not unconditional, which in $\mathbb{R}^{2}$ implies (AS). Hence $K_{0}$ is D-universal by Theorem 3.6, and therefore $K$ is D-universal by Proposititon 2.5. Necessity is clear since only unconditional convex bodies are generated by unconditional $K$.

Corollary 3.9. A generalised zonoid $K$ in $\mathbb{R}^{3}$ such that $F\left(K, e_{i}\right), i=1,2,3$, are singletons is D-universal if and only if none of the two-dimensional projections of $K$ is unconditional.

Example 3.10. In four dimensions consider convex body $K=\sum_{s \in \mathcal{S}^{4}} a_{s} s I$. If

$$
\begin{aligned}
& a_{(1,1,1,1)}=1, \quad a_{(-1,-1,1,1)}=2, \quad a_{(-1,1,-1,1)}=20, \quad a_{(-1,1,1,-1)}=24, \\
& a_{(-1,1,1,1)}=18, \quad a_{(1,-1,1,1)}=17, \quad a_{(1,1,-1,1)}=4, \quad a_{(1,1,1,-1)}=8 \text {, }
\end{aligned}
$$

then all six two-dimensional projections of $K$ satisfy the asymmetry condition (in particular, $K$ is not unconditional), whereas the four-dimensional case of condition (AS) fails.

\subsection{Transformation of surface area measures}

Let $S_{n-1}(L, \cdot)$ be the surface area measure of a symmetric convex body $L$, see [13, Sec. 4.2]. The $K$-transform of $S_{n-1}(L, \cdot)$ is the support function given by

$$
\int_{\mathbb{S}^{n-1}} h(u K, v) S_{n-1}(L, d v)=n V(L, \ldots, L, u K), \quad u \in \mathbb{R}^{n}
$$

where $V(L, \ldots, L, u K)$ is the mixed volume of $L$ and $u K$. The injectivity of $T_{K}$ implies that the values $V(L, \ldots, L, u K)$ for $u \in \mathbb{R}^{n}$ uniquely determine the set $L$. Theorems 3.2 and 3.6 imply the following fact.

Corollary 3.11. If $K$ is a generalised zonoid such that all support sets $F\left(K, e_{i}\right), i=$ $1, \ldots, n$, are singletons, then each unconditional convex body $L$ is uniquely determined by the values $V(L, \ldots, L, u K), u \in \mathbb{R}^{n}$. A general origin symmetric convex body $L$ is uniquely determined if $K$ satisfies the condition of Theorem 3.6.

If $K=I$ from (1.3), then $V(L, \ldots, L, u K)$ as function of $u$ is the support function of the projection body of $L$, see [13, Sec. 5.3.2]. For a general $K$, one obtains a generalisation of the projection body transform, so that $V(L, \ldots, L, u K), u \in \mathbb{R}^{n}$, is the support function of a convex body called the $K$-transform of $L$.

Example 3.12. Let $L=B$ be the unit Euclidean ball. Then $V(B, \ldots, B, u K)$ is proportional to the mean width of $u K$. The $K$-transform of the unit ball has the support function

$$
\frac{1}{n} \int_{\mathbb{S}^{n-1}} h(u K, v) d v, \quad u \in \mathbb{R}^{n}
$$




\section{Diagonally transformed $\ell_{p}$-balls and one-sided sta- ble laws}

\section{1 $\quad D_{p}$-balls}

For a symmetric closed convex set $L$, the Minkowski functional is defined by

$$
\|u\|_{L}=\inf \{t>0: u \in t L\}, \quad u \in \mathbb{R}^{n} .
$$

If $L$ is a symmetric convex body with non-empty interior, then $\|u\|_{L}$ is a norm with the unit ball being $L$. Let $\|x\|_{p}$ be the $p$-norm of $x$ with $p \in[1, \infty]$, and let $B_{p}=\left\{x:\|x\|_{p} \leq 1\right\}$ be the $\ell_{p}$-ball in $\mathbb{R}^{n}$.

Definition 4.1 . Let $\mu$ be a finite measure on $\mathbb{S}^{n-1}$, and let $p \in[1, \infty]$. The convex body $L$ with the Minkowski functional

$$
\|u\|_{L}=\int_{\mathbb{S}^{n-1}}\|u v\|_{p} \mu(d v), \quad u \in \mathbb{R}^{n}
$$

is called a $D_{p}$-ball. The measure $\mu$ is called the spectral measure of $L$.

In particular, $B_{p}$ is a $D_{p}$-ball with the spectral measure being the Dirac measure at $(1, \ldots, 1)$. Obviously, $D_{p}$-balls are unconditional for each $p$ and $\mu$. The measure $\mu$ in Definition 4.1 can always be chosen either unconditional or supported by $\mathbb{S}_{+}^{n-1}=$ $\mathbb{S}^{n-1} \cap \mathbb{R}_{+}^{n}$. Since $\mu$ is finite, all $D_{p}$-balls have non-empty interior. Moreover $L$ is bounded if and only if $\mu$ is not supported by a coordinate hyperplane.

It is easy to identify $\|u\|_{L}$ from (4.1) with $\left(T_{K} \mu\right)(u)$ for $K=B_{q}$ with $1 / p+1 / q=1$. Furthermore, (4.1) can be expressed as

$$
\|u\|_{L}=\mathbf{E}\|u \eta\|_{p}
$$

using an integrable random vector $\eta$. The polar body $L^{\circ}$ of the $D_{p}$-ball $L$ is a diagonal body generated by $B_{q}$ since

$$
h\left(L^{\circ}, u\right)=\mathbf{E}\|u \eta\|_{p}=\mathbf{E} h\left(u B_{q}, \eta\right)=\mathbf{E} h\left(\eta B_{q}, u\right), \quad u \in \mathbb{R}^{n} .
$$

For instance, the polar body of a $D_{2}$-ball is derived from diagonal transformations of the Euclidean ball, see Example 1.3. Note that $L$ is unbounded if and only if $L^{\circ}$ lies in a coordinate hyperplane.

For $u \in \mathbb{R}_{+}^{n}$ and $x \in \mathbb{R}$ we use the notation $u^{x}=\left(u_{1}^{x}, \ldots, u_{n}^{x}\right)$.

Example 4.2 ( $D_{1}$-balls). If $p=1$, then

$$
\int_{\mathbb{S}^{n-1}}\|u v\|_{1} \mu(d v)=\left\langle\int_{\mathbb{S}^{n-1}}|v| \mu(d v),|u|\right\rangle=\|u w\|_{1}=\|u\|_{w^{-1} B_{1}}
$$

whence each $D_{1}$-ball can be obtained as $w^{-1} B_{1}$ for some $w \in \mathbb{R}_{+}^{n}$. A component $w_{j}$ is zero if and only if $\mu$ is supported by the coordinate hyperplane perpendicular to $e_{j}$. In this case the $D_{1}$-ball is unbounded in the $e_{j}$-direction.

Remark 4.3. Definition 4.1 can be extended to $p \in(0,1)$, or to averages of arbitrary diagonally transformed norms on $\mathbb{R}^{n}$; this results in a (not necessarily convex) star-shaped set $L$. Furthermore, it is possible to introduce an $L^{r}$-variant of $D_{p^{-}}$-ball with $r \in[1, \infty)$ by considering $\left(\mathbf{E}\|u \eta\|_{p}^{r}\right)^{1 / r}$, that is, the $\mathrm{L}^{r}$-norm of $\|u \eta\|_{p}$. We may also allow $\mu$ to be a signed measure, however restricted by the requirement that the right-hand side of (4.1) is non-negative for all $u$. 


\subsection{One-sided strictly stable random vectors}

In the following we show that $D_{p}$-balls naturally appear in relation to one-sided stable laws if $p \in[1, \infty)$ and to max-stable laws if $p=\infty$. A random vector $\xi$ in $\mathbb{R}^{n}$ is strictly $\alpha$-stable if

$$
(t+s)^{1 / \alpha} \xi \stackrel{d}{=} t^{1 / \alpha} \xi^{\prime}+s^{1 / \alpha} \xi^{\prime \prime}
$$

for all $t, s>0$, where $\xi^{\prime}$ and $\xi^{\prime \prime}$ are independent copies of $\xi$ and $\stackrel{d}{=}$ denotes the equality in distribution. The parameter $\alpha$ is called characteristic exponent. The geometric interpretation of symmetric stable random vectors is worked out in [6]. In this case, $\alpha \in(0,2]$ (where $\alpha=2$ means that $\xi$ is Gaussian), and the characteristic function of $\xi$ can be written as

$$
\mathbf{E e}^{\imath\langle\xi, u\rangle}=\exp \left\{-\|u\|_{F}^{\alpha}\right\}, \quad u \in \mathbb{R}^{n},
$$

where $F$ is an $\mathrm{L}_{\alpha}$-ball, that is,

$$
\|u\|_{F}^{\alpha}=\int_{\mathbb{S}^{n-1}}|\langle u, v\rangle|^{\alpha} \sigma(d v)
$$

for a finite measure $\sigma$ on the unit sphere.

If $\xi$ is strictly $\alpha$-stable and $\xi \in \mathbb{R}_{+}^{n}$ a.s., then $\xi$ is said to be one-sided or totally skewed to the right. In this case $\alpha \in(0,1], \alpha=1$ identifies a deterministic $\xi$, and the Laplace transform of $\xi$ is given by

$$
\mathbf{E e}^{-\langle\xi, u\rangle}=\exp \left\{-\int_{\mathbb{S}_{+}^{n-1}}\langle u, v\rangle^{\alpha} \sigma(d v)\right\}, \quad u \in \mathbb{R}_{+}^{n},
$$

where the spectral measure $\sigma$ is finite and supported by $\mathbb{S}_{+}^{n-1}$. This follows, e.g., by applying general results of [2] to the semigroup $\mathbb{R}_{+}^{n}$ with the usual addition and identical involution.

Theorem 4.4. A non-trivial random vector $\xi$ in $\mathbb{R}_{+}^{n}$ is strictly $\alpha$-stable with $\alpha \in(0,1]$ if and only if there exists a $D_{1 / \alpha}$-ball $L$ such that the Laplace transform of $\xi$ is given by

$$
\mathbf{E e}^{-\langle\xi, u\rangle}=\exp \left\{-\left\|u^{\alpha}\right\|_{L}\right\}, \quad u \in \mathbb{R}_{+}^{n} .
$$

Proof. Sufficiency is immediate, since the Laplace transform of $t^{1 / \alpha} \xi$ is $\exp \left\{-t\left\|u^{\alpha}\right\|_{L}\right\}$, whence (4.2) holds. To prove necessity, if $\alpha=1$, then $\xi=x$ is deterministic, and $L=x^{-1} B_{1}$ is a $D_{1}$-ball. Now assume that $\alpha \in(0,1)$ and $\xi$ satisfies (4.4). Let $\tilde{\eta}$ be distributed on $\mathbb{S}_{+}^{n-1}$ according to the normalised $\sigma$, and let $\eta=\sigma\left(\mathbb{S}_{+}^{n-1}\right)^{1 / \alpha} \tilde{\eta}$, so that

$$
\int_{\mathbb{S}_{+}^{n-1}}\langle u, v\rangle^{\alpha} \sigma(d v)=\mathbf{E}(\langle u, \eta\rangle)^{\alpha}=\mathbf{E}\left\|u^{\alpha} \eta^{\alpha}\right\|_{1 / \alpha}=\left\|u^{\alpha}\right\|_{L}, \quad u \in \mathbb{R}_{+}^{n},
$$

where $L$ is the $D_{1 / \alpha}$-ball generated by the random vector $\eta^{\alpha}$.

Remark 4.5. If the arithmetic sum operation on the right-hand side of (4.2) is replaced by the coordinatewise maximum, $\xi$ is said to be max-stable. In this case, $\alpha$ can take an arbitrary positive value. It is shown in [5] that the cumulative distribution functions of max-stable random vectors with $\alpha=1$ are characterised as

$$
\mathbf{P}\{\xi \leq u\}=\exp \left\{-\left\|u^{-1}\right\|_{L}\right\}, \quad u \in(0, \infty)^{n},
$$


for a $D_{\infty}$-ball $L$. The polar set $L^{\circ}$ is a diagonal body generated by the $\ell_{1}$-ball in $\mathbb{R}^{n}$; such convex bodies $L^{\circ}$ were called max-zonoids in [5].

Theorem 4.4 is supplemented by the following existence result.

Lemma 4.6. For each $D_{1 / \alpha}$-ball $L$ with $\alpha \in(0,1]$ there is a strictly $\alpha$-stable random vector $\xi$ in $\mathbb{R}_{+}^{n}$ such that the Laplace transform of $\xi$ is given by (4.5).

Proof. We may assume $\alpha \in(0,1)$, the case $\alpha=1$ being obvious. Let $\hat{\eta}$ be a random vector in $\mathbb{R}_{+}^{n}$ such that $\mathbf{E}\|u \hat{\eta}\|_{1 / \alpha}=\|u\|_{L}$ for $u \in \mathbb{R}_{+}^{n}$. Define $\eta=\hat{\eta}^{1 / \alpha}$. Then

$$
\left\|u^{\alpha}\right\|_{L}=\mathbf{E}\left\|u^{\alpha} \eta^{\alpha}\right\|_{1 / \alpha}=\mathbf{E}(\langle u, \eta\rangle)^{\alpha}, \quad u \in \mathbb{R}_{+}^{n} .
$$

Define a finite measure $\sigma(A)=\mathbf{E}\left(\|\eta\|^{\alpha} \mathbb{1}_{\eta /\|\eta\| \in A}\right)$ for all Borel $A \subset \mathbb{S}_{+}^{n-1}$. Then

$$
\int_{\mathbb{S}_{+}^{n-1}}\langle u, v\rangle^{\alpha} \sigma(d v)=\mathbf{E}(\langle u, \eta\rangle)^{\alpha}, \quad u \in \mathbb{R}_{+}^{n}
$$

Thus it is enough to show that for each $\alpha \in(0,1)$ and each finite measure $\sigma$ there is a one-sided strictly $\alpha$-stable random vector $\xi$ such that (4.4) holds. Choose a sequence of measures $\sigma_{m}=\sum_{j=1}^{k_{m}} a_{m j} \delta_{v_{m j}}$ with $a_{m j}>0, v_{m j} \in \mathbb{S}_{+}^{n-1}, j=1, \ldots, k_{m}$, and $k_{m} \rightarrow \infty$, such that $\sigma_{m} \rightarrow \sigma$ weakly as $m \rightarrow \infty$. For each $m$ and $j$ define an independent $\alpha$ stable random vector $\xi_{m j}$ in $\mathbb{R}_{+}^{n}$ with completely dependend components having Laplace transform $\exp \left(-a_{m j}\left\langle u, v_{m j}\right\rangle^{\alpha}\right)$. Clearly the Laplace transform of the sum $\chi_{m}=\sum_{j=1}^{k_{m}} \xi_{m j}$ is

$$
\mathbf{E} e^{-\left\langle u, \chi_{m}\right\rangle}=\exp \left\{-\int_{\mathbb{S}_{+}^{n-1}}\langle u, v\rangle^{\alpha} \sigma_{m}(d v)\right\}, \quad u \in \mathbb{R}_{+}^{n} .
$$

By the pointwise convergence of Laplace transforms, the limit in distribution of $\chi_{m}$ exists and has the Laplace transform (4.6), see for example [4, Th. 5.22]. From its Laplace transform it is also obvious that the limit of $\chi_{m}$ is strictly $\alpha$-stable.

Example 4.7 (Completely dependent components). If the spectral measure is the Dirac measure at $v \in \mathbb{R}_{+}^{n}$, the corresponding $D_{p}$-ball is $v^{-1} B_{p}$ for $p \in[1, \infty)$. The corresponding one-sided $\alpha$-stable random vector with $\alpha=1 / p$ satisfies

$$
\mathbf{E e}^{-\langle\xi, u\rangle}=\mathrm{e}^{-\left\|v u^{\alpha}\right\|_{p}}=\exp \left\{-\left(u_{1} v_{1}^{1 / \alpha}+\cdots+u_{n} v_{n}^{1 / \alpha}\right)^{\alpha}\right\}, \quad u \in \mathbb{R}_{+}^{n} .
$$

This is the Laplace transform of the random vector $\zeta v^{1 / \alpha}$ obtained by scaling all components of $v^{1 / \alpha}$ with a one-sided strictly $\alpha$-stable random variable $\zeta$. In other words, the components of $\xi$ are completely dependent.

Example 4.8 (Independent components). If the components of one-sided strictly stable $\xi$ are independent, then

$$
\mathbf{E}^{-\langle\xi, u\rangle}=\exp \left\{-\left(v_{1} u_{1}^{\alpha}+\cdots+v_{n} u_{n}^{\alpha}\right)\right\}=\exp \left\{-\left\|u^{\alpha}\right\|_{v^{-1} B_{1}}\right\}, \quad u \in \mathbb{R}_{+}^{n},
$$

for some $v \in \mathbb{R}_{+}^{n}$. It follows that $B_{1}$ and thus any $D_{1}$-ball is a $D_{p}$-ball for each $p \in(1, \infty)$. 
For a closed convex set $L$ and $\beta>0$, define its signed $\beta$-power by

$$
L^{\langle\beta\rangle}=\left\{x^{\langle\beta\rangle}: x \in L\right\},
$$

where $x^{\langle\beta\rangle}$ is the vector composed of the signed powers of the components of $x$.

Lemma 4.9. If $L$ is a $D_{p}$-ball with $p \in[1, \infty)$ and $\beta \in(0,1)$, then $L^{\langle\beta\rangle}$ is a $D_{p / \beta}$-ball.

Proof. Let $\xi$ be one-sided stable in $\mathbb{R}_{+}^{n}$ with characteristic exponent $\alpha=1 / p$ such that (4.5) holds. Further let $\zeta$ be a one-sided stable random variable with characteristic exponent $\beta \in(0,1)$ and Laplace transform $\exp \left(-s^{\beta}\right), s \geq 0$. Then $\zeta^{1 / \alpha} \xi$ is also one-sided stable (also called sub-stable, see [11]) with characteristic exponent $\alpha \beta$, and

$$
\mathbf{E} \mathrm{e}^{-\left\langle\zeta^{1 / \alpha} \xi, u\right\rangle}=\exp \left\{-\left\|u^{\alpha}\right\|_{L}^{\beta}\right\}=\exp \left\{-\left\|u^{\alpha \beta}\right\|_{L^{\prime}}\right\}
$$

where $L^{\prime}$ correspond to $\zeta^{1 / \alpha} \xi$, i.e. $L^{\prime}$ is a $D_{p^{\prime}}$-ball with $p^{\prime}=1 /(\alpha \beta)=p / \beta$. Then

$$
\begin{aligned}
\|u\|_{L^{\prime}}=\left\|u^{1 / \beta}\right\|_{L}^{\beta}=\inf \left\{s>0: u^{1 / \beta} / s \in L\right\}^{\beta} & =\inf \left\{t>0:(u / t)^{1 / \beta} \in L\right\} \\
& =\|u\|_{L^{\langle\beta\rangle}} .
\end{aligned}
$$

It follows that $L^{\prime}=L^{\langle\beta\rangle}$.

Example 4.10. If $B_{p}$ is the $\ell_{p}$-ball and so is a $D_{p}$-ball, then $B_{p}^{\langle p / r\rangle}=B_{r}$. For $r>p$, this corresponds to the conclusion of Lemma 4.9.

Lemma 4.11. Let $L$ be a symmetric convex body, let $\zeta$ be an integrable random vector in $\mathbb{R}^{n}$, and define $K=\mathbf{E}(\zeta L)$. For $j \in\{1, \ldots, n\}$, if the support set $F\left(K, e_{j}\right)$ is a singleton, then $\mathbf{P}\left(\zeta_{j}=0\right)=0$. The converse implication holds if $F\left(L, e_{j}\right)$ is a singleton.

Proof. The first statement is clear. Now assume that $F\left(L, e_{j}\right)=\{y\}$ for some $j \in$ $\{1, \ldots, n\}$ and $y \in L$ and that $\mathbf{P}\left(\zeta_{j}=0\right)=0$. Then the derivative of $h(L, u)$ at $e_{j}$ in direction $x \in \mathbb{R}^{n}$ is $h_{L}^{\prime}\left(e_{j} ; x\right)=h\left(F\left(L, e_{j}\right), x\right)=\langle y, x\rangle$, see [13, Th. 1.7.2]. Then

$$
\begin{aligned}
h_{K}^{\prime}\left(e_{j} ; x\right) & =\lim _{\varepsilon \downarrow 0} \mathbf{E} h\left(\zeta L, e_{j}+\varepsilon x\right)=\lim _{\varepsilon \downarrow 0} \mathbf{E} h\left(L, \zeta_{j} e_{j}+\varepsilon \zeta x\right) \\
& =\lim _{\varepsilon \downarrow 0} \mathbf{E}\left(\left|\zeta_{j}\right| h\left(L, e_{j}+\varepsilon \zeta_{j}^{-1} \zeta x\right)\right) \\
& =\mathbf{E}\left(\left|\zeta_{j}\right| h_{L}^{\prime}\left(e_{j} ; \zeta_{j}^{-1} \zeta x\right)\right)=\left\langle y \mathbf{E}\left(\zeta \operatorname{sign}\left(\zeta_{j}\right)\right), x\right\rangle,
\end{aligned}
$$

confirming the linearity of $h_{K}^{\prime}\left(e_{j} ; x\right)$ in $x$, and so the second claim by [13, Cor. 1.7.3].

Theorem 4.12. If $L$ is a $D_{p}$-ball for some $p \in[1, \infty)$, then $L$ is a $D_{r}$-ball for $r \in(p, \infty]$.

Proof. First consider $r<\infty$. It suffices to prove the claim for $L=B_{p}$. The case $p=1$ is shown in Example 4.8. If $p>1$, then $B_{p}=B_{1}^{\langle 1 / p\rangle}$. Since $B_{1}$ is a $D_{s}$-ball for $s>1, B_{p}$ is a $D_{s p}$-ball by Lemma 4.9.

Now consider $r=\infty$. Since $L$ is a $D_{s}$-ball for all $s \in[p, \infty)$, for each $s \in[p, \infty)$ there is a finite measure $\mu_{s}$ on $\mathbb{S}_{+}^{n-1}$ such that

$$
\|u\|_{L}=\int_{\mathbb{S}_{+}^{n-1}}\|u v\|_{s} \mu_{s}(d v), \quad u \in \mathbb{R}^{n} .
$$


We have $\|v\|_{s} \geq\|v\|_{\infty}>c$ for $v \in \mathbb{S}_{+}^{n-1}$ and some $c>0$. Thus

$$
\|(1, \ldots, 1)\|_{L}=\int_{\mathbb{S}_{+}^{n-1}}\|v\|_{s} \mu_{s}(d v) \geq c \mu_{s}\left(\mathbb{S}_{+}^{n-1}\right) .
$$

This shows that $\left\{\mu_{s}\left(\mathbb{S}_{+}^{n-1}\right): s \in[p, \infty)\right\}$ is bounded. Now choose a sequence of numbers $r_{k} \rightarrow \infty, k \geq 1$, and random vectors $\xi^{(k)}=\mu_{r_{k}}\left(\mathbb{S}_{+}^{n-1}\right) \eta^{(k)}$ where $\eta^{(k)}$ is distributed as normalised $\mu_{r_{k}}$. Then $\left\{\xi^{(k)}, k \geq 1\right\}$ is a tight sequence, and

$$
\|u\|_{L}=\mathbf{E}\left\|u \xi^{(k)}\right\|_{r_{k}}, \quad u \in \mathbb{R}^{n} .
$$

Without loss of generality assume that $\xi^{(k)} \rightarrow \xi$ almost surely as $k \rightarrow \infty$ for some random vector $\xi$. By bounded convergence $\mathbf{E}\left\|u \xi^{(k)}\right\|_{r_{k}} \rightarrow \mathbf{E}\|u \xi\|_{\infty}$ for $u \in \mathbb{R}^{n}$. Thus, $L$ is a $D_{\infty}$-ball.

Corollary 4.13. If $L$ is a $D_{p}$-ball for some $p \in[1,2]$, then $L^{\circ}$ is a zonoid.

Proof. Since the Euclidean ball $B_{2}$ is a zonoid, the polar set of each $D_{2}$-ball is a zonoid. For $p \in[1,2)$ use Theorem 4.12 ,

\subsection{Unconditional D-universality of $\ell_{p}$-balls}

Given that $\|u \xi\|_{p}=h\left(\xi B_{q}, u\right)$, the following results show that $\ell_{q}$-balls are unconditionally D-universal for all $q \in[1, \infty)$ that is, $\mathbf{E}\|u \xi\|_{p}, u \in \mathbb{R}^{n}$, identify the distribution of $|\xi|$ up to zonoid equivalence. Recall that this is not true for $q=\infty$, see Example 1.2. For $q \in[2, \infty), B_{q}$ is a zonoid whose support sets satisfy the conditions of Theorem 3.2 , whence $B_{q}$ is unconditionally D-universal. However, $B_{q}$ is not necessarily a zonoid if $n \geq 3, q \in[1,2)$, e.g. if $q$ is close to one.

Theorem 4.14. Let $K$ be a symmetric convex body such that all support sets $F\left(K, e_{i}\right)$, $i=1, \ldots, n$, are singletons, and such that $K^{\circ}$ is a $D_{p}$-ball for some $p \in(1, \infty]$. Then $K$ is unconditionally D-universal.

Proof. By Theorem 4.12 we may assume that $p=\infty$. Now assume that (1.8) holds for $K=\mathbf{E}\left(\zeta B_{1}\right)$ where $\zeta$ is a random vector in $\mathbb{R}_{+}^{n}$ independent of $\xi$ and $\eta$. This implies

$$
\mathbf{E}\|u \zeta \xi\|_{\infty}=\mathbf{E}\|u \zeta \eta\|_{\infty}, \quad u \in \mathbb{R}^{n} .
$$

By Theorem 1.4,

$$
\mathbf{E}|\langle u, \zeta|\xi|\rangle|=\mathbf{E}|\langle u, \zeta|\eta|\rangle|, \quad u \in \mathbb{R}^{n}
$$

Now let $L=\mathbf{E}[-\zeta, \zeta]$ so that

$$
\mathbf{E} h(u L,|\xi|)=\mathbf{E} h(u L,|\eta|), \quad u \in \mathbb{R}^{n} .
$$

Since the support sets of the zonoid $L$ in coordinate directions are singletons by Lemma 4.11, the claim follows from Theorem 3.2 .

Corollary 4.15. If $\xi$ and $\eta$ are integrable random vectors in $\mathbb{R}_{+}^{n}$ and $p \in(1, \infty]$, then

$$
\mathbf{E}\|u \xi\|_{p}=\mathbf{E}\|u \eta\|_{p}, \quad u \in \mathbb{R}^{n}
$$

if and only if $\xi$ and $\eta$ are zonoid equivalent. 
Proof. In Theorem 4.14 set $K=B_{q}$ with $1 / p+1 / q=1$.

In the case of strictly positive $\xi$ and $\eta$, an analytical proof of the equivalence in Corollary 4.15 is given in [8]. Note that the case $p=1$ is excluded (see Example 1.2); it would correspond to $K=B_{\infty}$ which does not satisfy the condition on support sets imposed in Theorem 4.14.

By Theorems 2.3 and 4.14, linear combinations of support functions of polar sets of $D_{p}$-balls are dense in the family of support functions of unconditional convex bodies if $p \in(1, \infty]$ and if the conditions on the support sets are satisfied.

\section{Appendix: General transformations}

For completeness, we mention the following result generalising Theorem 2.1 to a more general subfamily $G$ of linear transformations on $\mathbb{R}^{n}$. The $G$-invariant Minkowski class generated by $K \in \mathscr{K}_{0}$ consists of the limits in the Hausdorff metric of the linear combinations

$$
\alpha_{1} g_{1} K+\cdots+\alpha_{m} g_{m} K
$$

where $\alpha_{1}, \ldots, \alpha_{m}>0, g_{1}, \ldots, g_{m} \in G$ and $m \geq 1$. Due to the presence of scaling constants $\alpha_{i}$, it is possible to assume that $G$ is bounded. Let $\nu$ be a finite signed measure on $G$ equipped with the Borel $\sigma$-algebra. Denote

$$
\left(T_{K, G} \nu\right)(u)=\int_{G} h(g K, u) \nu(d g), \quad u \in \mathbb{R}^{n} .
$$

The dominated convergence theorem yields that $T_{K, G} \nu$ is continuous. Unlike the case of diagonal transformations, it is not possible to swap $g$ and $u$ in $h(g K, u)$. The following result can be derived following the same arguments as in [13, Th. 3.5.3].

Proposition A.1. A convex body L belongs to the $G$-invariant Minkowski class generated by $K$ if and only if $h(L, u)=\left(T_{K, G} \nu\right)(u)$ for a finite even measure $\nu$ on $G$.

Theorem A.2. The linear space spanned by the support functions from the G-invariant Minkowski class generated by $K$ is dense in the family of support functions of convex bodies from $\mathscr{K}_{0}$ if and only if the transform

$$
\mu \mapsto \int_{\mathbb{S}^{n-1}} h(g K, v) \mu(d v), \quad g \in G
$$

is injective for finite even signed measures $\mu$ on $\mathbb{S}^{n-1}$.

Proof. Necessity. The support function $|\langle u, v\rangle|$ of the segment $u I$ can be approximated by linear combinations of the support functions $h\left(g_{i} K, v\right)$. Hence, if the right-hand side of (A.1) vanishes for all $g \in G$, the cosine transform of $\mu$ vanishes. So the transform (A.1) is injective.

Sufficiency. Denote by $\mathscr{M}_{G}$ the family of finite signed measures on $G$ with the total variation norm. The operator $T_{K, G}$ is continuous and maps measures from $\mathscr{M}_{G}$ to continuous even functions on the unit sphere. Its adjoint $T_{K, G}^{\prime}$ is an operator on the family 
$\mathscr{M}_{e}$ of signed finite even measures on $\mathbb{S}^{n-1}$, and $T_{K, G}^{\prime} \mu$ belongs to the dual space of $\mathscr{M}_{G}$. For $\mu \in \mathscr{M}_{e}$ and $\nu \in \mathscr{M}_{G}$,

$$
\left\langle T_{K, G}^{\prime} \mu, \nu\right\rangle=\left\langle\mu, T_{K, G} \nu\right\rangle=\int_{\mathbb{S}^{n-1}}\left(T_{K, G} \nu\right) d \mu,
$$

where the left-hand side refers to the pairing of $T_{K, G}^{\prime} \mu$ and $\nu$. If $T_{K, G}^{\prime} \mu=0$, then

$$
\int_{\mathbb{S}^{n-1}} \int_{G} h(g K, u) \nu(d g) \mu(d u)=0
$$

for all $\nu \in \mathscr{M}_{G}$. Changing the order of integration yields that

$$
\int_{\mathbb{S}^{n-1}} h(g K, u) \mu(d u)=0
$$

whence $\mu=0$ by injectivity. The triviality of the kernel of $T_{K, G}^{\prime}$ yields that the range of $T_{K, G}$ is dense in the space of continuous functions on $\mathbb{S}^{n-1}$, see [17, Th. III.4.5].

If $G$ is the group of all invertible linear transformations, then [1, Th. 5(1)] yields that, for each symmetric convex body $K$, the equality $\mathbf{E} h(g K, \xi)=\mathbf{E} h(g K, \eta), g \in G$, holds if and only if $\xi$ and $\eta$ are zonoid equivalent.

Similar results hold in the unconditional case meaning that the linear combinations of support functions of convex bodies from the $G$-invariant Minkowski class are dense in the family of support functions of convex bodies from $\mathscr{K}_{s}$ if and only if $\mathbf{E} h(g K, \xi)=\mathbf{E} h(g K, \eta)$ for all $g \in G$ yields that $|\xi|$ and $|\eta|$ are zonoid equivalent. Note that, for general $G$, the Minkowski class may contain convex bodies that are not in $\mathscr{K}_{s}$ though $K$ is from $\mathscr{K}_{s}$. In the special case of diagonal transformations this does not occur.

\section{Acknowledgement}

This work was supported by the Swiss National Science Foundation Grant 200021_153597.

\section{References}

[1] S. Alesker. On $\mathrm{GL}_{n}(\mathbb{R})$-invariant classes of convex bodies. Mathematika, 50:57-62, 2003.

[2] Yu. Davydov, I. Molchanov, and S. Zuyev. Strictly stable distributions on convex cones. Electron. J. Probab., 13:259-321, 2008.

[3] Z. Kabluchko, M. Schlather, and L. de Haan. Stationary max-stable fields associated to negative definite functions. Ann. Probab., 37:2042-2065, 2009.

[4] O. Kallenberg. Foundations of Modern Probability. Springer, New York, 2nd edition, 2002.

[5] I. Molchanov. Convex geometry of max-stable distributions. Extremes, 11:235-259, 2008. 
[6] I. Molchanov. Convex and star shaped sets associated with multivariate stable distributions. I. Moments and densities. J. Multivariate Anal., 100:2195-2213, 2009.

[7] I. Molchanov. Theory of Random Sets. Springer, London, 2nd edition, 2017.

[8] I. Molchanov and M. Schmutz. Multiasset derivatives and joint distributions of asset prices. In M. Rutkowski Yu. Kabanov and T. Zariphopoulou, editors, Inspired by Finance. The Musiela Festschrift. Springer, Berlin, 2014.

[9] I. Molchanov, M. Schmutz, and K. Stucki. Invariance properties of random vectors and stochastic processes based on the zonoid concept. Bernoulli, 20:1210-1233, 2014.

[10] K. Mosler. Multivariate Dispersion, Central Regions and Depth. The Lift Zonoid Approach, volume 165 of Lect. Notes Statist. Springer, Berlin, 2002.

[11] G. Samorodnitsky and M. S. Taqqu. Stable non-Gaussian Random Processes. Chapman \& Hall, New York, 1994.

[12] R. Schneider. Equivariant endomorphisms of the space of convex bodies. Trans. Amer. Math. Soc., 194:53-78, 1974.

[13] R. Schneider. Convex Bodies. The Brunn-Minkowski Theory. Cambridge University Press, Cambridge, 2nd edition, 2014.

[14] R. Schneider and F. E. Schuster. Rotation invariant Minkowski classes of convex bodies. Mathematika, 54:1-13, 2007.

[15] R. A. Vitale. Expected absolute random determinants and zonoids. Ann. Appl. Probab., 1:293-300, 1991.

[16] Y. Wang and S. Stoev. On the association of sum- and max-stable processes. Statist. Probab. Lett., 80:480-488, 2010.

[17] D. Werner. Funktionalanalysis. Springer-Verlag, Berlin, 3rd edition, 2000. 